\title{
Studies on the Growth and Micro hardness, Dielectrics and Photoconductivity of NLO Material Ammonium acid phthalate
}

\author{
Suresh Sagadevan \\ Department of Physics, \\ Sree Sastha Institute of \\ Engineering and Technology, \\ Chembarambakkam, \\ Chennai-600 123, \\ India
}

\author{
R.Varatharajan \\ Department of Mechanical Engineering, \\ Sree Sastha Institute of Engineering and \\ Technology, Chembarambakkam, \\ Chennai-600 123, India
}

\begin{abstract}
Single crystals of Ammonium acid phthalate have been grown using the slow evaporation technique. A single crystal of good quality was harvested in a period of 30 days. Different characterization studies were carried out for finding its suitability in device fabrications. The lattice parameters were found from single crystal X-ray diffraction analysis. The grown crystal crystallized under orthorhombic system with Pcab space group. The Vicker's microhardness studies were performed to understand the mechanical behavior of crystals. The dielectric studies were carried on the grown crystals to study the electric behavior of these crystals. Photoconductivity measurements carried out on the grown crystal reveal the negative photoconducting nature
\end{abstract}

\section{Keywords}

Single X-ray diffraction, Vickers microhardness, Non-linear optical materials, Dielectric constant, Dielectric loss and Photoconductivity studies.

\section{INTRODUCTION}

In the emerging modern technologies, a lot of efforts were taken to find new higher order nonlinear optical materials for various optoelectronic applications. This technology is continuing to establish and the improvement in the development of lasers and higher nonlinear optical materials have resulted in a variety of commercially available nonlinear optical devices [1]. Among nonlinear optical (NLO) materials, organic materials have involved greater attention due to their large optical susceptibilities, inherent ultrafast response times and high damage resistance when compared to other materials [2]. The search for new materials with high optical nonlinearity is important for both academic and industrial purposes. These materials have concerned the interests of many theoretical and experimental researchers. In semi organic materials, the organic ligand is ionically bonded with the inorganic host therefore the semi-organic crystals have higher mechanical strength and chemical stability [3]. The present investigation deals with the growth of ammonium acid phthalate single crystals by slow solvent evaporation technique. The grown crystals have been subjected to single XRD analysis, Vickers hardness test, dielectric measurements and photoconductivity studies. The results of these studies are discussed.

\section{EXPERIMENTAL PROCEDURES}

Ammonium acid phthalate single crystal was synthesized from ammonia and phthalic acid in the molar ratio 1:1. A saturated solution of ammonium acid phthalate was prepared at room temperature. The solution was kept in a petri dish for a period of seven days. The ammonium acid phthalate crystals were recrystallised many times to minimize the impurity content. Normally, recrystallization process tends to minimize the defects and impurities. A saturated solution was prepared from the recrystallised ammonium acid phthalate salt. Then the solution was filtered using a Whatmann filter paper and transferred to a beaker covered with airtight thick filter paper, so that the rate of evaporation can be minimised. Fine seed crystals were obtained over a period of 10 days. These seed crystals were harvested into crystals by keeping in the mother solution over a period of 30 days. The photograph of the as grown crystal is shown in Fig.1. 


\section{RESULTS AND DISCUSSION}

\subsection{Single Crystal X-Ray Diffraction}

Single crystal X-ray diffraction studies for the grown crystals were carried out to determine the lattice parameters of the ammonium acid phthalate crystals.Ammonium acid phthalate crystallizes in the orthorhombic system having space group Pcab. The unit cell parameters obtained are $\mathrm{a}=6.31 \AA$, $\mathrm{b}=10.08 \AA, \mathrm{c}=25.73 \AA, \alpha=\gamma=\beta=90^{\circ}$. The structure of the grown crystal has been confirmed by single-crystal XRD which is very good agreement with the reported values [4]. The space group predicted reveals the non centrosymmetric nature of the grown crystal

\subsection{Microhardness studies}

The mechanical properties of the ammonium acid phthalate crystals were performed using Vickers hardness test at room temperature. The hardness of a material is a measure of its resistance to deformation. Normally, permanent deformation is achieved by indentation, bending, scratching and cutting. In an ideal crystal, completely free from defects, the hardness is independent of the applied load. The hardness measurements were carried for a load of 10 to $50 \mathrm{~g}$. The Vicker's hardness number $\left(H_{v}\right)$ of the ammonium acid phthalate is calculated using the relation

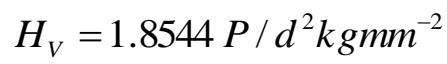

where $\mathrm{P}$ is the applied load in $\mathrm{Kg}$ and $\mathrm{d}$ is the diagonal length of the impression in $\mathrm{mm}$. The values of $\mathrm{Hv}$ for various loads are plotted and shown in Fig.2. It is evident that the Vickers hardness number decreases with increase in load satisfying the indentation size effect. By plotting the applied load vs Vicker's hardness number, the value of the work hardening coefficient of ammonium acid phthalate was found to be 2.15 , which indicates that it belongs to the soft category. According to Onitsch [5] ' $\mathrm{n}$ ' lies between 1 and 1.6 for hard materials and is greater than 1.6 for soft materials $[7,6]$. The ' $n$ ' value observed in the present studies is around 2.15 suggesting that the grown ammonium acid phthalate crystal is a relatively softer material.

The soft nature has been predicted from the investigations of microhardness and the mechanical behavior of ammonium acid phthalate is found to be similar to that of reported values [4]. The soft nature of the material ammonium acid phthalate crystal is revealed from the work hardening coefficient value 2.15 which is in good agreement with the reported value 1.9 by Arunkumar et al [4] for the similar compound of ammonium acid phthalate. Even though the material has larger NLO efficiency, the material possesses lower damage threshold due to soft nature.

\subsection{Dielectric Properties}

The dielectric constant and the dielectric loss were determined as a function of frequency and temperatures. The cut and polished single crystals of ammonium acid phthalate were used for dielectric studies. The surface of the sample was electroded with silver paste for electrical contact. The experiment was carried out at frequencies of $50 \mathrm{~Hz}$ to $5 \mathrm{MHz}$ at different temperatures of $30{ }^{\circ} \mathrm{C}$ to $90{ }^{\circ} \mathrm{C}$, respectively. The variation of the dielectric constant of the grown crystal as a function of frequency is shown in Fig.3. From the Fig.3, the dielectric constant is seen to decrease with an increase in the frequency. It is found that the dielectric constant and the dielectric loss generally increase with increase in temperature.
The high value of dielectric constant in the low frequency region may be due to the contributions from all four polarizations, namely, electronic, ionic, orientation and space charge polarizations [8]. The electronic exchange of the number of ions in the crystals gives local displacement of electron in the direction of the applied field, which in turn give rise to polarization. The decrease in the value of the dielectric constant with frequency is due to the fact that the frequency of electric charge carriers cannot follow the alternation of the ac electric field applied, beyond a certain critical frequency [9]. The very low value of the dielectric constant at higher frequencies is significant for the fabrication of materials for ferroelectric, photonic and electro-optic devices. The variation of dielectric loss with frequency is shown in Fig. 4. These curves suggest that the dielectric loss depends on the frequency of the applied field, similar to what commonly happens with the dielectric constant in the ionic system $[10,11]$. The dielectric loss decreases with an increase in the frequency at almost all temperatures but appears to attain saturation in the higher frequency and above at all temperatures. The characteristic of low dielectric loss with high frequency for a given crystal suggests that the crystal possesses good optical quality with lesser defects and this parameter is of vital importance for nonlinear optical materials in their applications [12].

It is concluded that the polarization (dielectric behavior) increases with decrease of frequency for almost all amino acid group of single crystals [13]. It is understood that the contribution of all types of polarization will be more at lower frequencies.

\subsection{Photoconductivity studies}

Photoconductivity was carried out using the Keithley 485 picoammeter. The dark current was recorded by keeping the sample unexposed to any radiation. Fig. 5 shows the variation of both the dark current $\left(\mathrm{I}_{\mathrm{d}}\right)$ and photocurrent $\left(\mathrm{I}_{\mathrm{p}}\right)$ with applied field. It is seen from the plots that both $I_{d}$ and $I_{p}$ of the sample increase linearly with applied field. It is experimental from the plot that the dark current is always higher than the photocurrent, thus confirming the negative photoconductivity nature of the grown crystal. The photocurrent is found to be less than the dark current, which is termed as negative photoconductivity. The phenomenon of negative photoconductivity is explained using the Stockmann model [14]. The negative photoconductivity in a solid is due to the decrease in the number of charge carriers or their lifetime, in the presence of radiation [15]. For a negative photoconductor, the forbidden gap contains two energy levels in which, one is situated between the Fermi level and the conduction band while the other is located close to the valence band. The second state has a higher capture cross-section for electrons and holes. As it captures electrons from the conduction band and holes from the valence band, the number of charge carriers in the conduction band gets reduced and the current decreases in the presence of radiation. The upper energy level is situated between the Fermi level and the conduction band, whereas the other one is located in the neighbourhood of the valence band. The lower level has high capture cross-section for electrons from the conduction band and holes from the valence band. As a result, when the sample is kept under exposed light, the recombination of electrons and holes takes place, resulting in decrease in the number of mobile charge carriers, giving rise to negative photoconductivity. The negative photoconductivity of the sample may be due to the reduction in the number of charge carriers to reveal the dielectric nature of the material. 


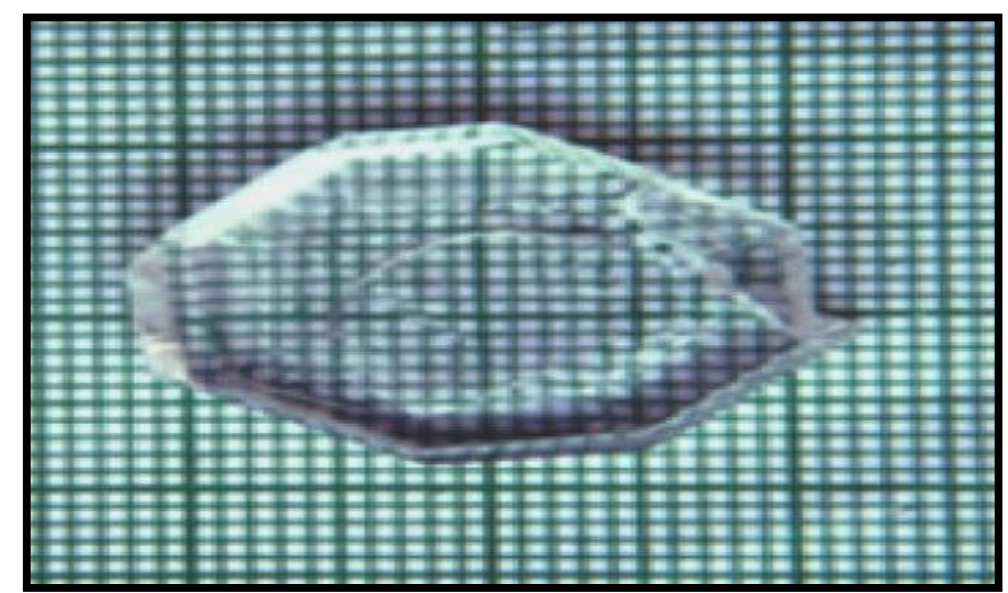

Fig.1. Photograph of as grown crystal of ammonium acid phthalate

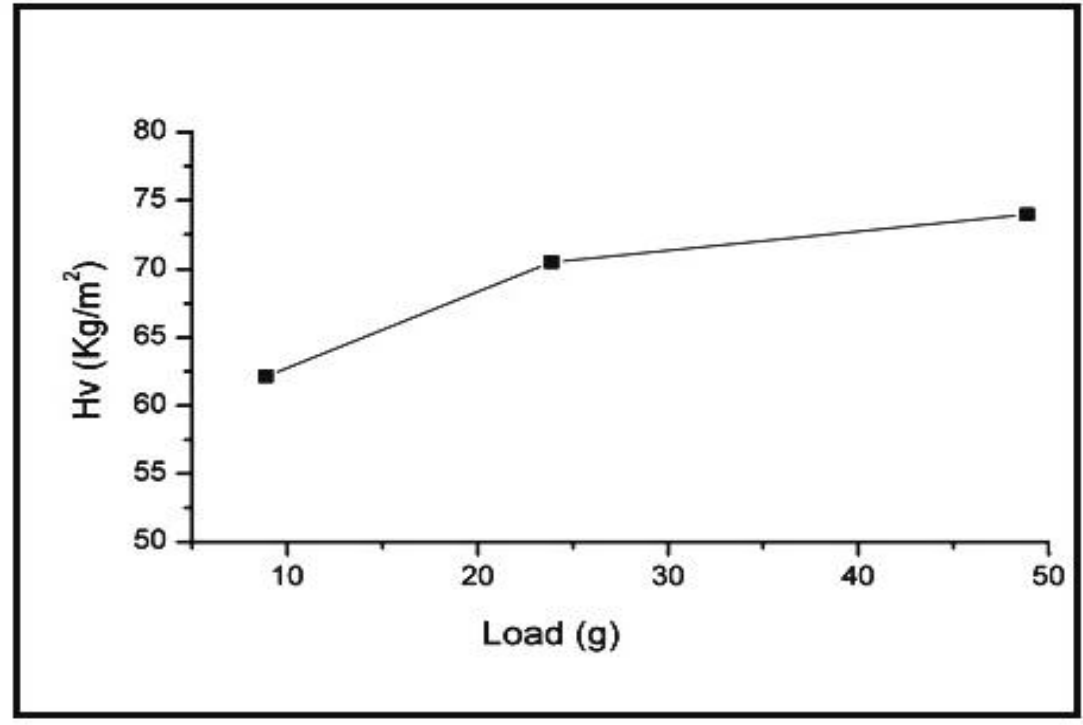

Fig.2 Plot of microhardness against load 


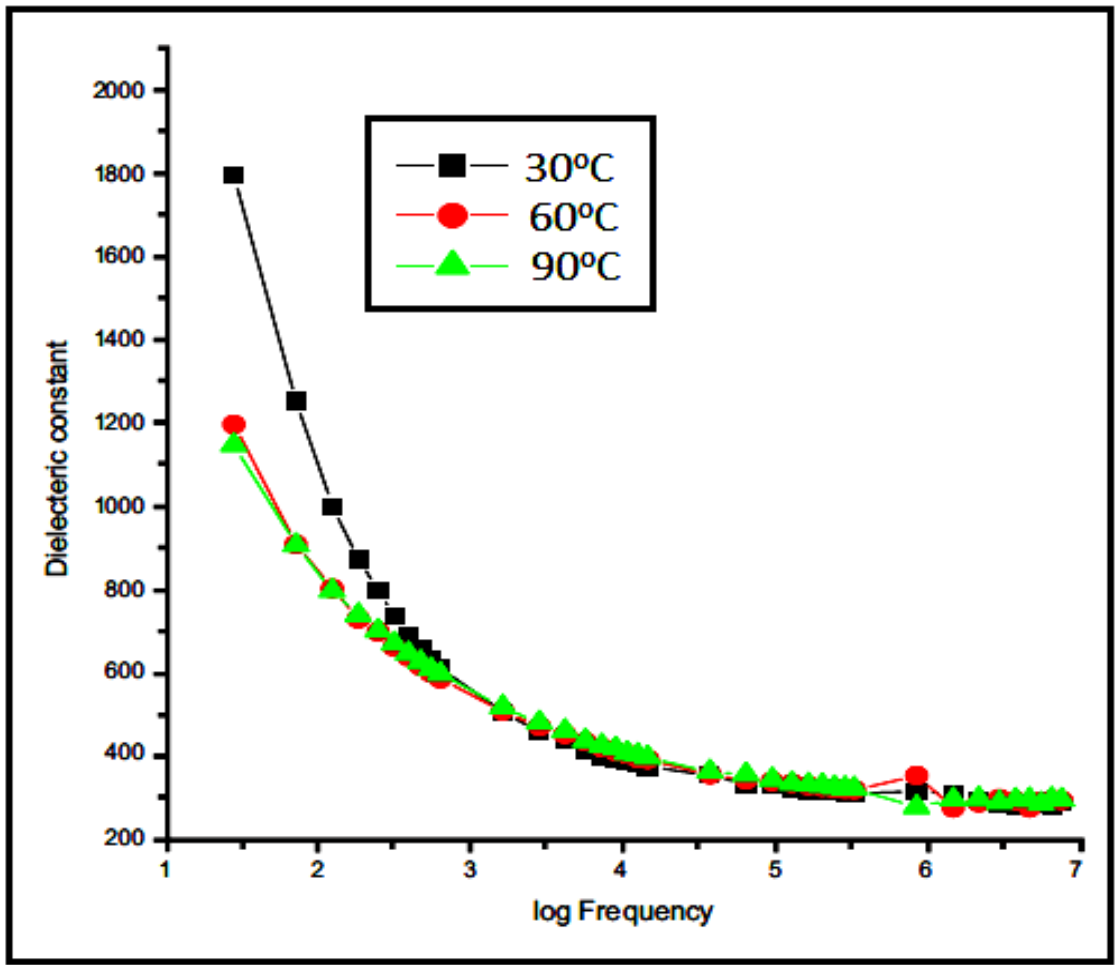

Fig.3 Variation of dielectric constant with frequency for ammonium acid phthalate crystal

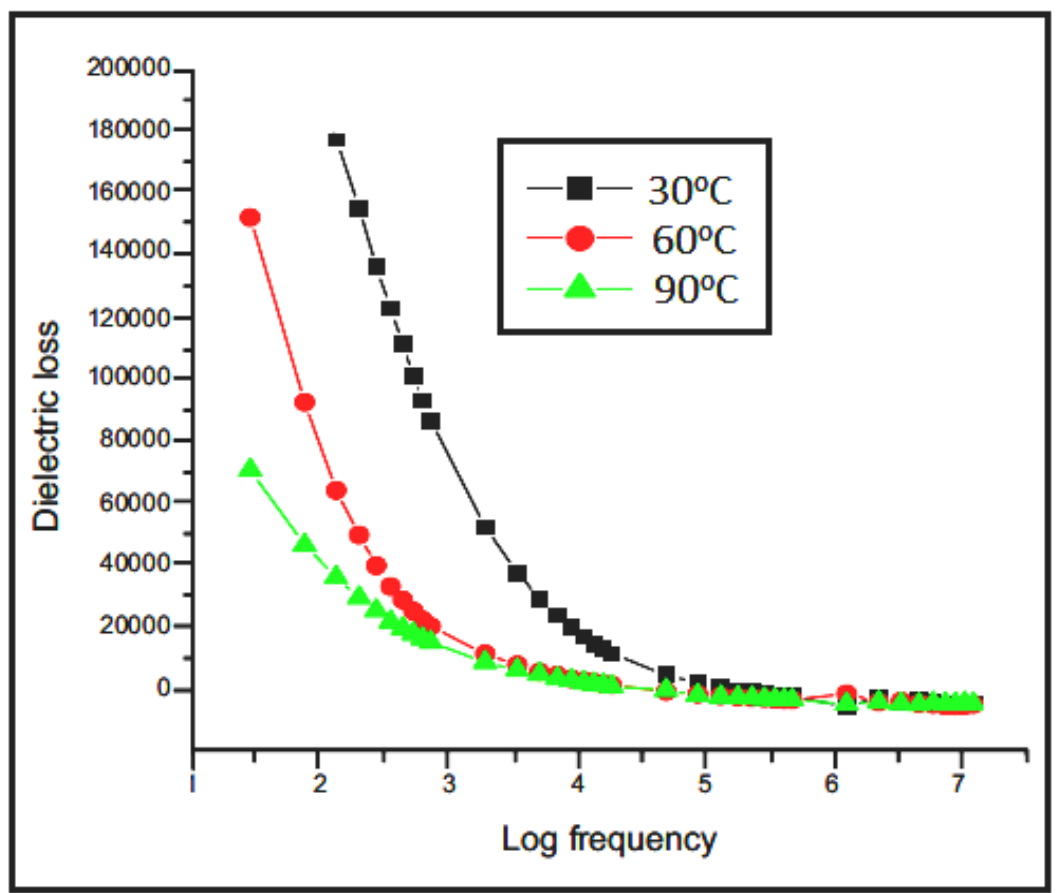

Fig.4. Variation of dielectric loss with frequency for ammonium acid phthalate crystal 


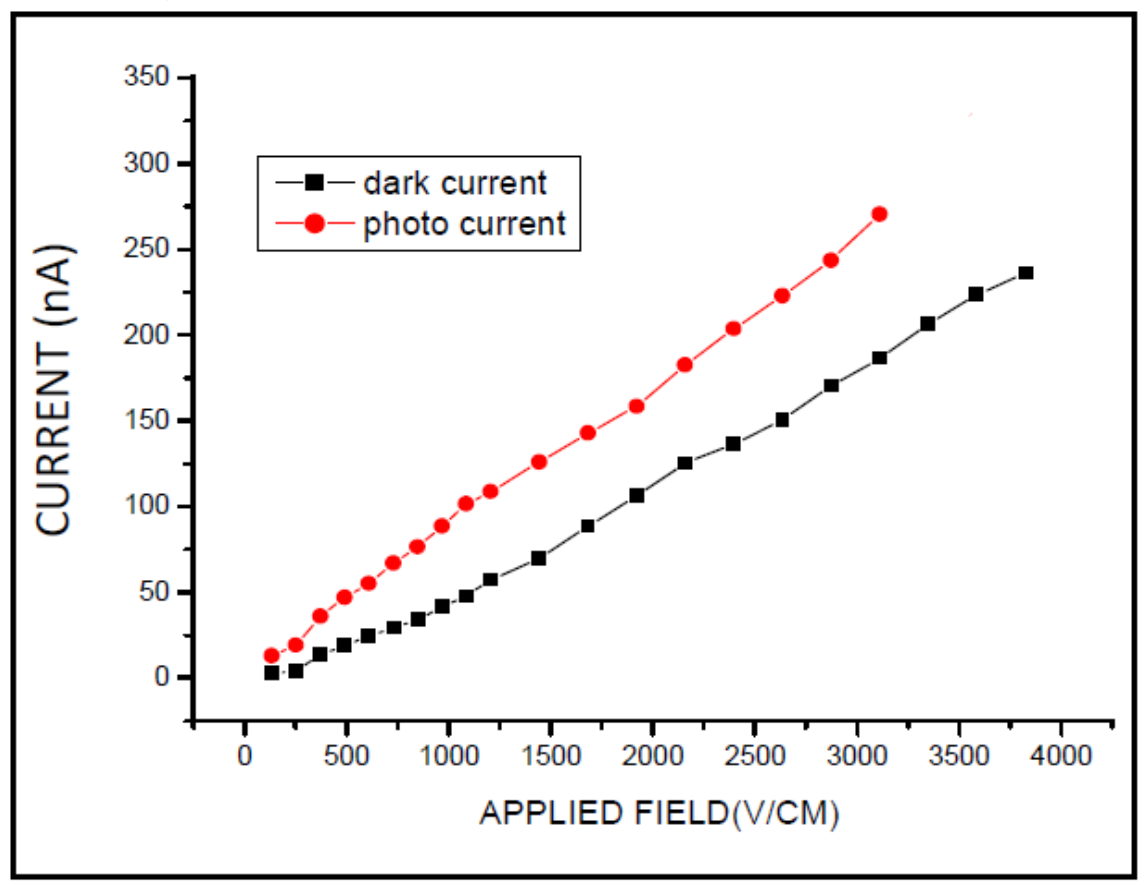

Fig.5 Field dependent photoconductivity of ammonium acid phthalate

\section{CONCLUSION}

The single crystals of ammonium acid phthalate were grown using the slow evaporation technique. Its lattice parameters have been found from single crystal XRD analysis. Vickers hardness values were determined inorder to study the mechanical properties of the crystal. The dielectric studies reveal the low dielectric constant and low dielectric loss of the crystals at high frequency range, which is ideal for NLO materials. This supports the applicability of crystals of high optical quality with lesser defects for NLO applications. The photoconductivity measurement confirms the negative photoconductivity nature of the grown crystal.

\section{ACKNOWLEDGEMENTS}

The authors thank the Management of Sree Sastha Institute of Engineering and Technology, Chembarambakkam, Chennai 600123 for their encouragements throughout this work. One of the authors S.Suresh , thank to R.Varatharajan, Principal, Sree Sastha Institute of Engineering and Technology, Chembarambakkam, Chennai -600123, for spending his precious time in scientific discussions, for his personal involvement, innovative ideas, and critical and valuable suggestions throughout the preparation of this paper.

\section{REFERENCES}

[1] Munn, R.W.; Ironside, C.N.; Chapman and Hall, London.1993

[2] Kaino, T.; Cai, B.; Takayama, K. Adv. Funct. Mater, 12 (2002) 599-603.
[3] Long, N.J.; Angew. Chem. Int. Ed. Engl, 1995, 34, 21-38.

[4] Arunkumar A, P. Ramasamy, P, Optical Materials, 2013

[5] Onitsch EM, Microscope, 1950, Vol. 95, pp. 12.

[6] Ramesh Babu R., Kumaresan S., Vijayan N., Gunasekaran M., Gopalakrishnan R., and Kannan P, J. Cryst Growth; 2003, Vol. 256, pp. 387-92

[7] Rajesh NP., Kannan V., Ashok M., Sivaji K., Santhana Raghavan P., Ramasamy P., J. Crystal Growth, 2004, Vol. 262, pp. 5616

[8] Bhat, S.I.; Rao P.M.; Bhat, A.P.G.; and Avasthi, D.K. Surface and coating Technology, 158(2002) 725-728.

[9] Ponpandian, N.; Balaya, P.; and Narayanasamy, A. J. Phys., Condens.Matter, 2002,14,3221.

[10]Rao, K.V.; and Smakula, A. J. Appl. Phys, 1966, 37,319323.

[11]Rao, K.V.; and Smakula, A. J. Appl. Phys, 1965, 36, 2031-2038.

[12] Balarew, C.; and Duhlew, R. J.Solid State Chem, 1984, 55, 1-6.

[13]Cyrac Peter A, Vimalan M, Sagayaraj P, and Madhavan J, Physica B: Condensed Matter, 2010, Vol. 405, pp. 65-71

[14] Joshi, V.N.; Photoconductivity, Marcel Dekker, New York.1990.

[15]Bube, R.H.; Photoconductivity of solids, Wiley, New York.1981. 